\title{
Salinomycin induces apoptosis and differentiation in human acute promyelocytic leukemia cells
}

\author{
YI ZHAO $^{1}$, LIANG ZHONG ${ }^{2}$, LU LIU $^{2}$, SHI-FEI YAO ${ }^{1}$, MIN CHEN $^{1}$, LIAN-WEN LI $^{1}$, \\ ZHI-LING SHAN $^{2}$, CHUN-LAN XIAO ${ }^{1}$, LIU-GEN GAN ${ }^{1}$, TING XU ${ }^{1}$ And BEI-ZHONG LIU ${ }^{1,2}$ \\ ${ }^{1}$ Central Laboratory of Yongchuan Hospital, Chongqing Medical University, Chongqing 402160; ${ }^{2}$ Key Laboratory of \\ Laboratory Medical Diagnostics, Ministry of Education, Department of Laboratory Medicine, \\ Chongqing Medical University, Chongqing 400016, P.R. China
}

Received July 8, 2017; Accepted June 12, 2018

DOI: $10.3892 /$ or.2018.6513

\begin{abstract}
At present, acute promyelocytic leukemia (APL) is the most curable form of acute myeloid leukemia and can be treated using all-trans retinoic acid and arsenic trioxide. However, the current treatment of APL is associated with some issues such as drug toxicity, resistance and relapse. Therefore, other strategies are necessary for APL treatment. In the present study, we investigated the effects of salinomycin (SAL) on APL cell lines NB4 and HL-60 and determined its possible mechanisms. We observed that SAL inhibited cell proliferation, as determined by performing Cell Counting Kit-8 (CCK-8) assay, promoted cell apoptosis, as determined based on morphological changes, and increased Annexin V/ propidium iodide (PI)-positive apoptotic cell percentage. Treatment with SAL increased Bax/Bcl-2 and cytochrome $c$ expression and activated caspase -3 and -9 , thus leading to poly(ADP-ribose) polymerase (PARP) cleavage and resulting in cell apoptosis. These results revealed that SAL induced cell apoptosis through activation of the intrinsic apoptosis pathway. The present study is the first to show that SAL induced the differentiation of APL cells, as determined based on mature morphological changes, increased NBT-positive cell and CD11b-positive cell percentages and increased $\mathrm{CD} 11 \mathrm{~b}$ and $\mathrm{C} / \mathrm{EBP} \beta$ levels. Furthermore, SAL decreased the expression of $\beta$-catenin and its targets cyclin D1 and C-myc. Results of immunofluorescence analysis revealed that SAL markedly decreased the $\beta$-catenin level in both the nucleus and cytoplasm. Combination treatment with SAL and IWR-1, an inhibitor of Wnt signaling, synergistically triggered SAL-induced differentiation of APL cells. These findings demonstrated that SAL effectively inhibited cell prolifera-
\end{abstract}

Correspondence to: Dr Bei-Zhong Liu, Central Laboratory of Yongchuan Hospital, Chongqing Medical University, 1 Medical Road, Chongqing 402160, P.R. China

E-mail: liubeizhong@cqmu.edu.cn

Key words: acute promyelocytic leukemia, salinomycin, proliferation, apoptosis, differentiation, Wnt/ $\beta$-catenin signaling tion accompanied by induction of apoptosis and promotion of cell differentiation by inhibiting Wnt/ $\beta$-catenin signaling. Collectively, these data revealed that SAL is a potential drug for treatment of APL.

\section{Introduction}

Acute promyelocytic leukemia (APL), a distinct subtype of acute myeloid leukemia (AML), is characterized by reciprocal chromosomal translocation of $\mathrm{t}(15 ; 17)$, which results in the production of promyelocytic leukemia-retinoic acid receptor $\alpha$ (PML-RAR $\alpha$ ) fusion protein (1). For decades, APL has been considered as the most malignant form of AML due to its severe bleeding tendency and high early mortality rate $(2,3)$. Notably, at present, APL is the most curable form of AML and can be treated using all-trans retinoic acid (ATRA) and arsenic trioxide (ATO), which mainly induce cell differentiation and apoptosis $(3,4)$. However, APL treatment is associated with some issues such as ATRA or ATO resistance, relapse, differentiation syndrome and adverse effects (5-7). Therefore, it is necessary to identify other therapeutic strategies for APL treatment.

Salinomycin (SAL), a polyether antibiotic, is widely used as an anticoccidial drug for poultry (8). Recently, Gupta et al performed high-throughput screening of 16,000 compounds and found that SAL selectively killed breast cancer stem cells (CSCs) at least 100-times more effectively than conventional chemotherapeutic drug paclitaxel (9). Further studies have indicated that SAL exerts potential anticancer effects against different human cancer cell types, including lung, gastric and prostate cancer, and glioblastoma cells (10-13), without adversely affecting healthy cells (14-16). Accumulating evidence suggests that the presence of CSCs, which have capability of self-renewal and tumor-initiating capacities, are the major cause of drug resistance and relapse after therapy (17). SAL affects the proliferation of various CSCs including those present in breast, gastric and ovarian cancer $(9,18,19)$. A previous study revealed that SAL treatment also reversed multidrug resistance in leukemia stem cells (LSCs) such as KG-1a cells (20). Moreover, SAL was revealed to reverse multi-drug resistance in many cancer cell types $(21,22)$. Collectively, these findings revealed that SAL 
is a potential anticancer drug. However, limited studies have assessed the effect of SAL against leukemia.

SAL is also known as an inhibitor of $\mathrm{Wnt} / \beta$-catenin signaling (23) which plays key roles in both normal cell development and tumorigenesis (24). Aberrant activation of $\mathrm{Wnt} / \beta$-catenin signaling is frequently implicated in the pathogenesis of AML. Notably, high $\beta$-catenin expression was observed in both AML cell lines and primary blasts $(25,26)$. In addition, recent studies have shown that $\mathrm{Wnt} / \beta$-catenin signaling was associated with leukemia cell differentiation. Attenuation of Wnt/ $\beta$-catenin signaling promoted cell differentiation (27), whereas its activation blocked monocytemacrophage differentiation in AML cell lines (28). Therefore, we hypothesized that SAL induced APL cell differentiation by blocking Wnt/ $\beta$-catenin signaling.

Since limited studies have assessed the cytotoxicity of SAL against leukemia cells and its effect on leukemia cell differentiation, we investigated the effect of SAL in APL cell lines NB4 and HL-60 in the present study. We found that SAL markedly inhibited cell proliferation and induced the apoptosis and differentiation of APL cell lines NB4 and HL-60. Our results provide a foundation for further exploring the clinical use of SAL.

\section{Materials and methods}

Materials. Salinomycin (HY-15597) and IWR-1 (HY-12238) were purchased from MedChem Express (Monmouth Junction, NJ, USA). Cell Counting Kit-8 (CCK-8) reagent was purchased from Sevenseas Futai Biotechnology Co., Ltd. (Shanghai, China). Hoechst 33258 reagent was purchased from Beyotime Institute of Biotechnology (Shanghai, China). Wright Giemsa stain solution was purchased from Beijing Solarbio Science \& Technology Co., Ltd. (Beijing, China). The phycoerythrin (PE)-conjugated CD11b antibody (1:20; cat. no. 301306) was purchased from BioLegend, Inc., (San Diego, CA, USA). The antibody against CD11b (1:1,000; cat. no. ab133357) was purchased from Abcam (Cambridge, MA, USA). The antibody against LRP6 (1:1,000; cat. no. sc-25317) was purchased from Santa Cruz Biotechnology, Inc. (Dallas, TX, USA). Antibodies against caspase-3 (1:1,000; cat. no. 9665),caspase-9 (1:1,000; cat. no. 9504), cleaved caspase-9 (1:1,000; cat. no. 9509), cytochrome $c(1: 1,000$; cat. no. 11940$), \beta$-catenin $(1: 1,000$; cat. no. 8480), cyclin D1 (1:1,000; cat. no. 2922) and C-myc (1:1,000; cat. no. 5605) were purchased from Cell Signaling Technology, Inc. (Danvers, MA, USA). Antibodies against Bax (1:500; cat. no. wl01637) and Bcl-2 (1:500; cat. no. w101158), PARP (1:500; cat. no. WL01932) were purchased from Wanleibio Co., Ltd. (Shenyang, China). Goat anti-rabbit secondary antibody (1:4,000; cat. no. ZB-2301), goat antimouse secondary antibody $(1: 4,000$; cat. no. ZB-2305) and anti- $\beta$-actin antibody $(1: 1,000$; cat. no. BM0627) were purchased from Zhongshan Golden Bridge Biotechnology; OriGene Technologies (Beijing, China).

Cell lines and culture. The human APL cell lines NB4 and HL-60 obtained from the Shanghai Institutes for Biological Sciences (Shanghai, China) were then maintained in our own laboratory and cultured in RPMI-1640 medium supplemented with $10 \%$ fetal bovine serum (FBS; both from Gibco; Life
Technologies, Carlsbad, CA, USA) and penicillin (100 mg/ml) and streptomycin $(100 \mathrm{mg} / \mathrm{ml})$ in an environment that contained $5 \% \mathrm{CO}_{2}$ at $37^{\circ} \mathrm{C}$.

Cell viability assay. NB4 or HL-60 cells were seeded into 96-well plates with RPMI-1640 medium supplemented with $10 \%$ FBS. For experimental purposes, the cells were seeded at a density of $1 \times 10^{4}$ cells/well, and then treated with different concentrations of SAL for 24,48 or $72 \mathrm{~h}$, respectively. Then, $10 \mu \mathrm{lCCK}-8$ reagent was added to each well. Following incubation for $2 \mathrm{~h}$, cell viability was assessed by detection of the absorbance at $450 \mathrm{~nm}$ using a spectrophotometer (Bio-Rad Laboratories, Inc., Hercules, CA, USA). The experiment was repeated at least three times.

Hoechst 33258 staining. Cells were treated with SAL for $48 \mathrm{~h}$. Cells were collected and washed twice using phosphate-buffered saline (PBS) and plated onto the glass slides. After being fixed with $4 \%$ paraformaldehyde for $20 \mathrm{~min}$, the cells were permeabilized with $0.1 \%$ Triton X-100 for $15 \mathrm{~min}$. Subsequently, the cells were stained with Hoechst 33258 reagent for $10 \mathrm{~min}$ at $37^{\circ} \mathrm{C}$. The slides were washed three times with PBS. Finally, the nuclear morphological changes were observed under a fluorescence microscope (magnification, x400).

Wright-Giemsa staining. After $72 \mathrm{~h}$ of treatment, cells were collected and washed with PBS three times. Then the cells were resuspended in PBS and fixed on slides. The morphological changes of the cells were examined by optical microscopy (magnification, x200 or x1,000) after staining with Wright-Giemsa stain solution.

NBT reduction assay. For the nitroblue tetrazolium (NBT) reduction assay, NB4 and HL-60 cells were treated with SAL $(0.6 \mu \mathrm{M})$ or ATRA $(1 \mu \mathrm{M}$, as a positive control) for 3 days. Then each cell suspension was mixed with an equal volume of RPMI-1640 medium containing $1 \mathrm{mg} / \mathrm{ml} \mathrm{NBT}$ (Sigma-Aldrich, St. Louis, MO, USA) and $200 \mathrm{ng} / \mathrm{ml}$ TPA (Sigma-Aldrich; Merck) for $30 \mathrm{~min}$ at $37^{\circ} \mathrm{C}$. A total of 200 cells were counted by optical microscope (magnification, x1,000) after staining with or without Wright-Giemsa stain solution

Western blot analysis. For protein analysis, harvested cells were washed with ice-cold phosphate-buffered saline (PBS) three times and lysed in RIPA solution containing protease inhibitor phenylmethanesulfonyl fluoride (PMSF), phosphatase inhibitor $\mathrm{NaF}$ and Na3VO3. Protein concentration was measured by BCA method. Equal amounts of extracted total protein (30 or $50 \mu \mathrm{g}$ ) were separated by $10 \%$ or $12 \%$ polyacrylamide gels and then transferred to polyvinylidene difluoride (PVDF) membranes (EMD Millipore, Billerica, MA, USA). The membranes were blocked with $5 \%$ skim milk for $2 \mathrm{~h}$ at room temperature, and then incubated with the primary antibodies $(1: 1,000$ or $1: 500)$ overnight at $4^{\circ} \mathrm{C}$. The membranes were then incubated with goat anti-rabbit or goat anti-mouse secondary antibodies $(1: 4,000)$ for $1 \mathrm{~h}$ at $37^{\circ} \mathrm{C}$. After washing with Tris-buffered saline containing Tween-20 (TBST), the immunoreactive complexes were visualized using an enhanced chemiluminescence system (GE Healthcare, Marlborough, MA, USA). $\beta$-actin was used 
A

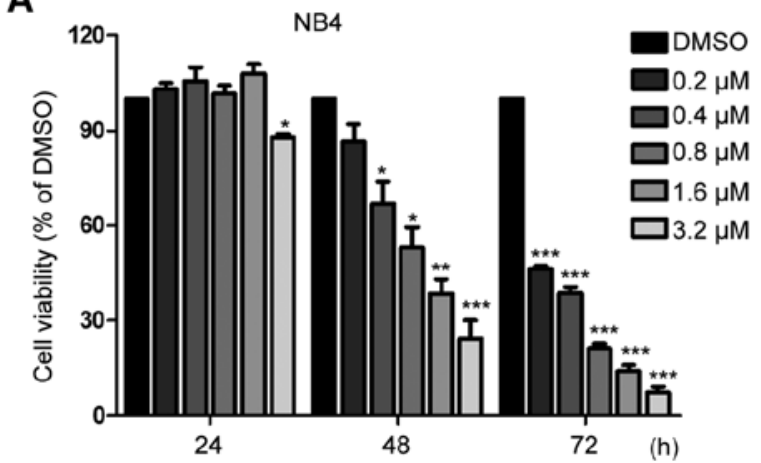

B

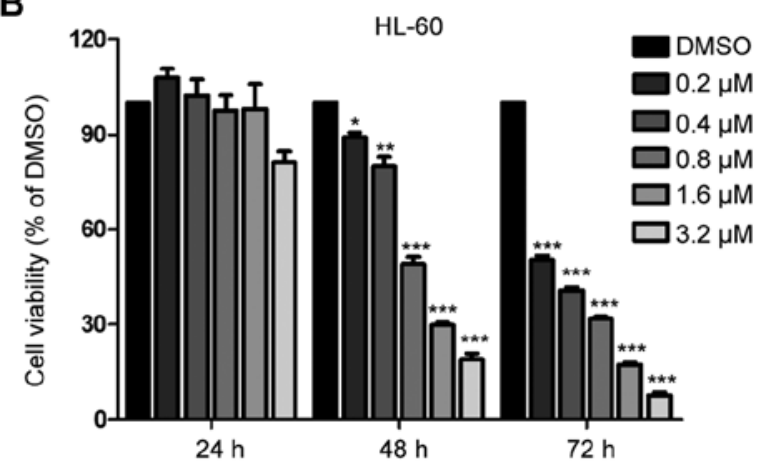

Figure 1. SAL inhibits the proliferation of APL cells. (A) NB4 and (B) HL-60 cells were cultured with SAL at the indicated dosages for 1, 2 and 3 days. Cell viability was detected by CCK- 8 assays. Data are expressed as the means $\pm \mathrm{SD}$ of three independent experiments. ${ }^{*} \mathrm{P}<0.05,{ }^{* *} \mathrm{P}<0.01,{ }^{* * * *} \mathrm{P}<0.001$ vs. the $\mathrm{DMSO}$ group, $\mathrm{n}=3$. SAL, salinomycin; APL, acute promyelocytic leukemia; CCK-8, Cell Counting Kit-8; DMSO, dimethyl sulphoxide.

as the internal positive control. Each experiment was repeated at least three times.

Flow cytometric assay. For apoptosis analysis, cells treated with different concentrations of SAL for $48 \mathrm{~h}$ were harvested and washed three times with pre-cold PBS. Cells were resuspended and stained with Annexin V-FITC and propidium iodide (PI) (Sigma-Aldrich; Merck KGaA, Darmstadt, Germany). The rate of cell apoptosis was analyzed using a FACSorter (BD Biosciences, San Jose, CA, USA) after incubation for $15 \mathrm{~min}$ at room temperature.

For the detection of cell surface differentiation marker CD11b, cells treated with SAL or ATRA for $72 \mathrm{~h}$ were washed three times with ice-cold PBS and then incubated with phycoerythrin (PE)-conjugated CD11b antibody at $4^{\circ} \mathrm{C}$ for $30 \mathrm{~min}$ in the dark. The cells were then washed three times with ice-cold PBS and then analyzed using flow cytometry (BD FACSVantage; BD Biosciences) and CellQuest Pro software version 5.1 (BD Pharmingen; BD Biosciemces, San Diego, CA, USA).

Indirect immunofluorescence assay. The localization of $\beta$-catenin was confirmed by indirect immunofluorescence assay. Cells were harvested, centrifuged at 1,000 x $\mathrm{g}$ for $5 \mathrm{~min}$ at room temperature and washed three times using PBS. Then, cells fixed with $4 \%$ paraformaldehyde for $20 \mathrm{~min}$ were permeabilized with $0.1 \%$ Triton $\mathrm{X}-100$ for $15 \mathrm{~min}$ and then blocked with $10 \%$ goat serum for $30 \mathrm{~min}$ at room temperature. The slides were incubated with the primary antibody $\beta$-catenin (1:200; cat. no. 8480$)$ at $4^{\circ} \mathrm{C}$ overnight. After being washed three times with PBS, the cells were incubated with secondary antibody goat against rabbit-IgGFITC (1:200; cat. no. ZF0311; Zhongshan Golden Bridge Biotechnology Co., Ltd.; OriGene Technologies) for $1 \mathrm{~h}$ at room temperature. Then nuclei were stained using DAPI (1:10; Beyotime Institute of Biotechnology) for $5 \mathrm{~min}$ at room temperature. Finally, the coverslips were viewed using a fluorescence microscope (magnification, x400) (Nikon Corp., Tokyo, Japan).

Statistical analysis. Statistical analysis was performed using SPSS 17.0 software (SPSS, Inc., Chicago, IL, USA). Data were expressed as the means \pm standard (SD). One-way analysis of variance followed by the Dunnett's test was performed and Student's t-test was used for comparisons. A value of $\mathrm{P}<0.05$ was considered to indicate a statistically significant result.

\section{Results}

Salinomycin inhibits the proliferation of APL cells. We performed CCK- 8 assay to determine the viability of NB4 and HL-60 cells which were treated with various concentrations $(0-3.2 \mu \mathrm{M})$ of SAL for 24,48 and $72 \mathrm{~h}$. SAL significantly inhibited cell viability in a dose-dependent manner after treatment for 48 and $72 \mathrm{~h}$ (Fig. 1). However, treatment with low concentrations $(0-1.6 \mu \mathrm{M})$ of SAL for $24 \mathrm{~h}$ did not exhibit cytotoxicity against NB4 (Fig. 1A) and HL-60 (Fig. 1B) cells. Therefore, we selected a time-point of $48 \mathrm{~h}$ to investigate the effect of SAL on cell apoptosis in subsequent experiments.

Salinomycin exerts a pro-apoptotic effect on NB4 and HL-60 cells. To examine whether SAL-induced cell death of NB4 and HL-60 cells was mediated by apoptosis induction, we performed Annexin V-FITC and PI staining and flow cytometric analysis. As shown in Fig. 2A, SAL treatment increased the percentage of apoptotic NB4 and HL-60 cells in a dose-dependent manner. The percentage of apoptotic NB4 cells increased from $6.01 \%$ among control cells [treated with dimethyl sulphoxide (DMSO)] to 32.30, 61.90 and $76.22 \%$ among cells treated with $0.8,1.6$ and $3.2 \mu \mathrm{M} \mathrm{SAL}$, respectively. The apoptotic HL- 60 cells increased from $3.16 \%$ among control cells (treated with DMSO) to 37.45, 64.46 and $85.75 \%$ among cells treated with $0.8,1.6$ and $3.2 \mu \mathrm{M}$ SAL, respectively. Results of Hoechst 33258 staining revealed altered morphology of NB4 and HL-60 cells treated with 1.6 $\mu \mathrm{M}$ SAL for $48 \mathrm{~h}$. SAL-treated cells exhibited typical morphological changes associated with apoptosis, such as nuclear fragmentation and condensation (Fig. 2B). To further explore the mechanism of apoptosis induced by SAL in NB4 and HL-60 cells we examined the expression levels of apoptosis-associated proteins including Bcl-2, Bax, caspase-3, -8 and -9, cleaved PARP and cytochrome $c$. As shown in Fig. 2C, SAL increased the expression level of Bax while it decreased the expression level of Bcl-2 and deregulated the ratio of Bax/ Bcl-2 in NB4 and HL-60 cells. Furthermore, the increased expression levels of cleaved caspase-3, cleaved caspase- 9 
A
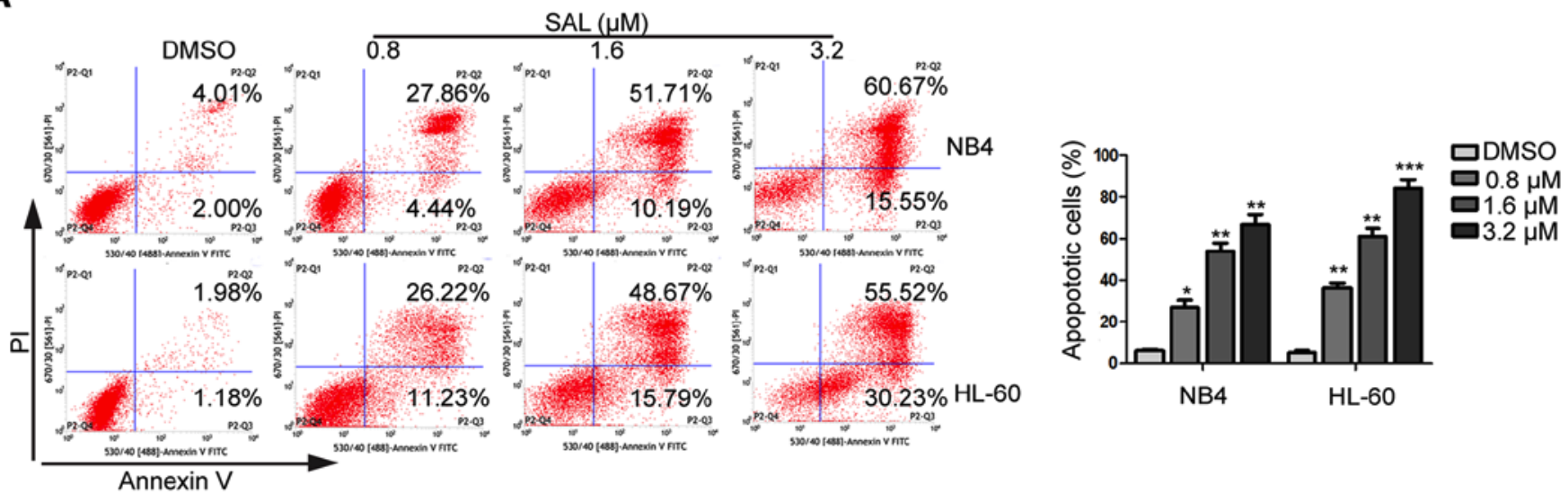

B

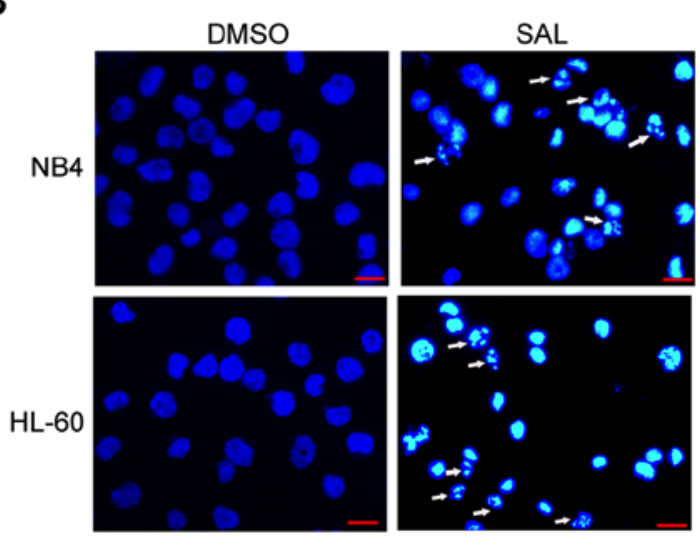

D

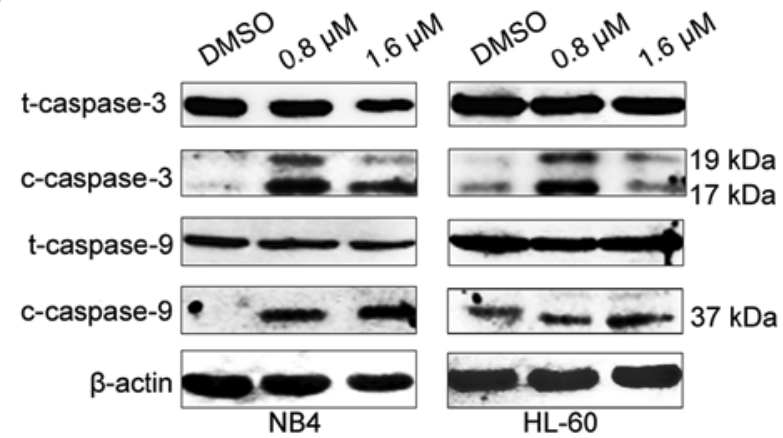

C

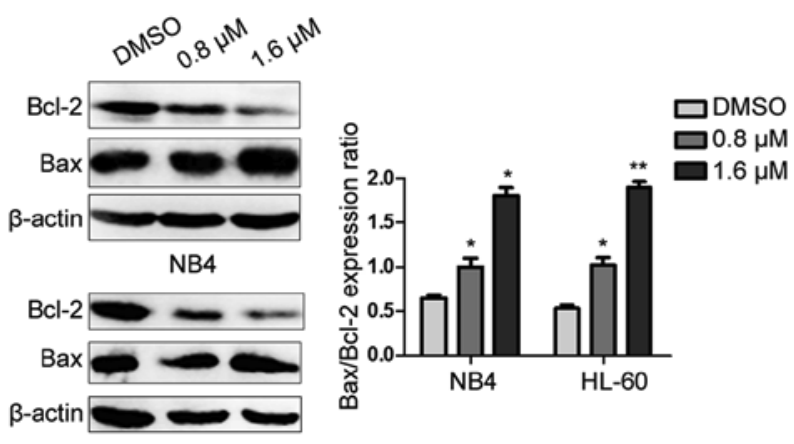

HL-60
$\mathbf{E}$

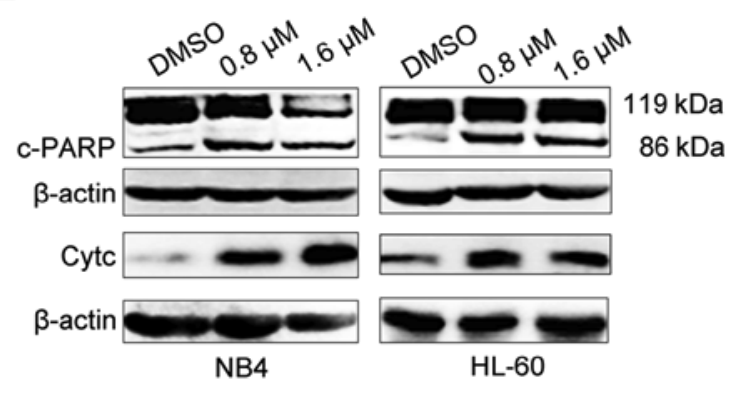

Figure 2. SAL has a pro-apoptotic effect on NB4 and HL-60 cells. (A) Cell apoptosis was assessed by Annexin V-FITC/propidium iodide (PI) staining assay by flow cytometry. The images display a representative experiment from three independent experiments. (B) Apoptotic cell morphology was assessed by Hoechst 33258 staining after treatment with the DMSO control or SAL $(1.6 \mu \mathrm{M})$ for $48 \mathrm{~h}$ (magnification, $\mathrm{x} 400)$. (C-E) NB4 and HL-60 cells were treated with indicated concentrations of SAL for $48 \mathrm{~h}$. (C) Then, the apoptosis-related protein levels of Bax and Bcl-2 were assessed by western blotting with the $\beta$-actin protein as an internal control. The relative protein expression levels of Bax/Bcl-2 ratio was quantified. Data are expressed as the means \pm SD of three independent experiments. (D) Caspase-3, cleaved-caspase-3, caspase-9 and cleaved caspase-9 levels were assessed by western blotting. (E) PARP and cytochrome $c$ levels were assessed by western blotting. ${ }^{*} \mathrm{P}<0.05,{ }^{* *} \mathrm{P}<0.01,{ }^{* * *} \mathrm{P}<0.001$ vs. the DMSO group, $\mathrm{n}=3$. SAL, salinomycin; DMSO, dimethyl sulphoxide.

(Fig. 2D) and cleaved PARP and cytochrome $c$ (Fig. 2E) were observed after SAL treatment. However, cleaved caspase-8 was not detected after SAL treatment (data not shown). Collectively, these data indicated that SAL effectively induced the apoptosis of APL cells.

Salinomycin induces the differentiation of APL cells. Targeting Wnt/ $\beta$-catenin signaling using 6-benzylthioinosine was revealed to induce the differentiation of leukemia cells (27). In addition, shRNA-mediated downregulation of $\beta$-catenin promoted ATRA-induced differentiation of HL-60 cells (29). Therefore, we determined whether SAL, a Wnt signaling inhibitor, also induced the differentiation of leukemia cells. For this, NB4 and HL-60 cells were incubated with SAL $(0.6 \mu \mathrm{M})$ or ATRA ( $1 \mu \mathrm{M}$, positive control) for $72 \mathrm{~h}$, and cell differentiation was evaluated based on morphological changes by performing 
A
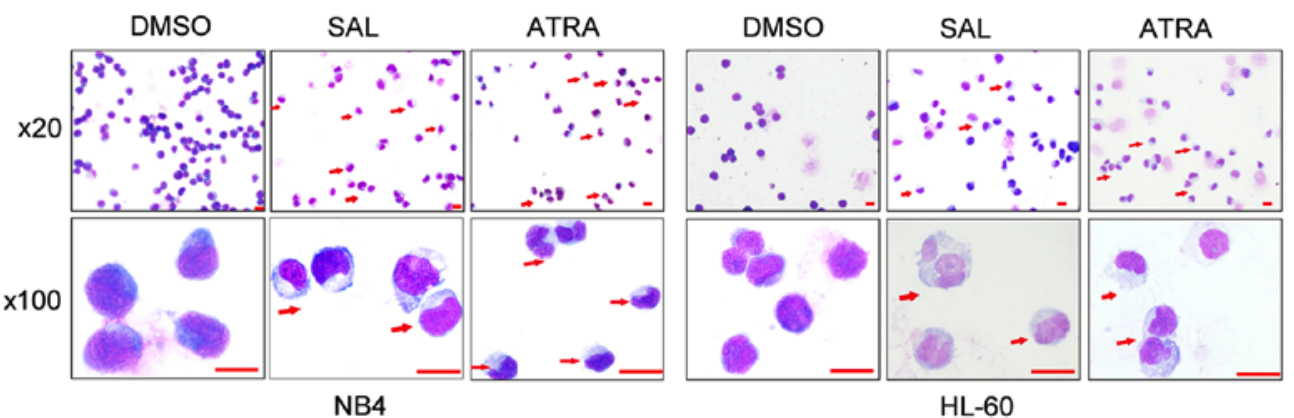

HL-60

B
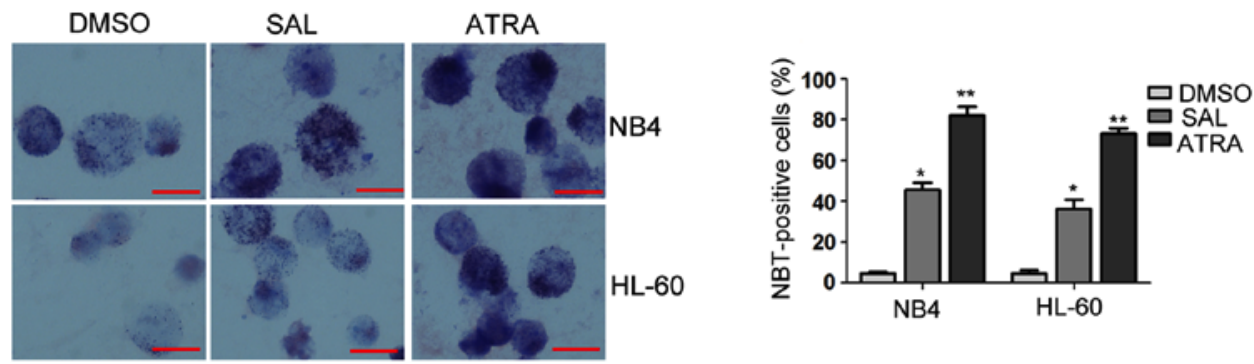

C
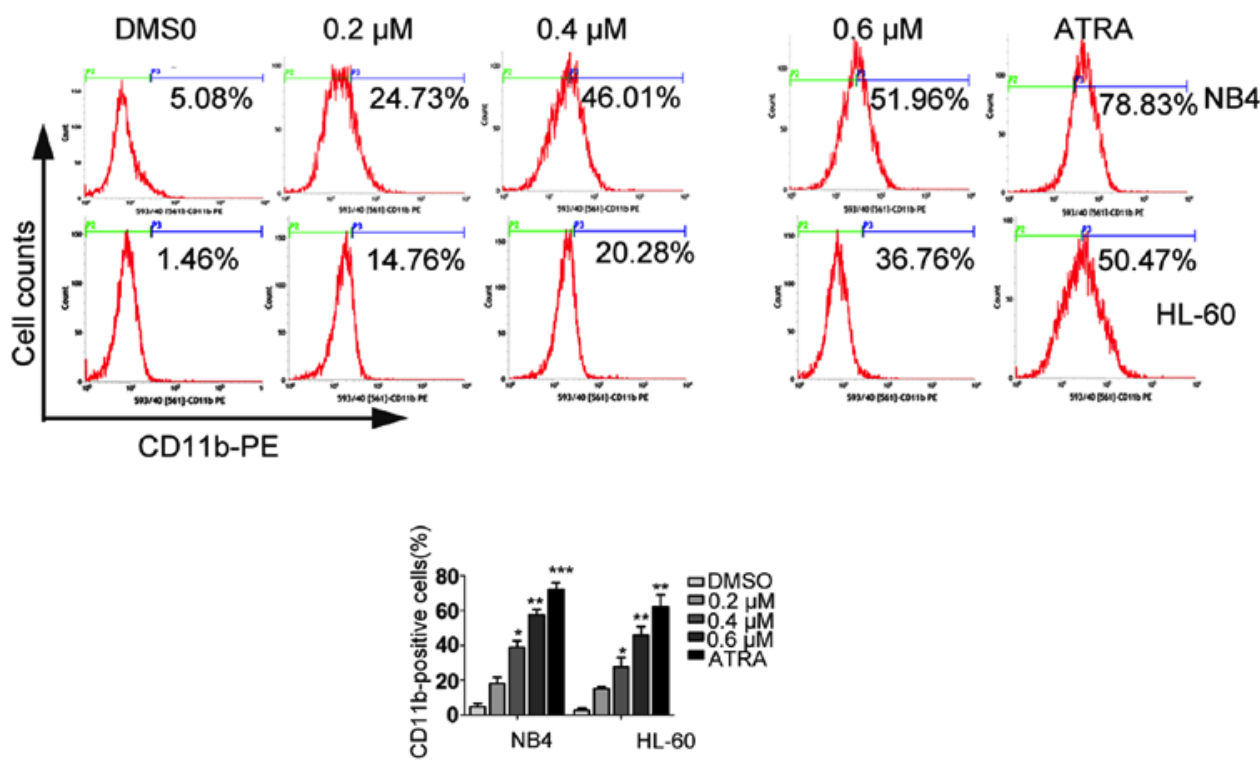

D
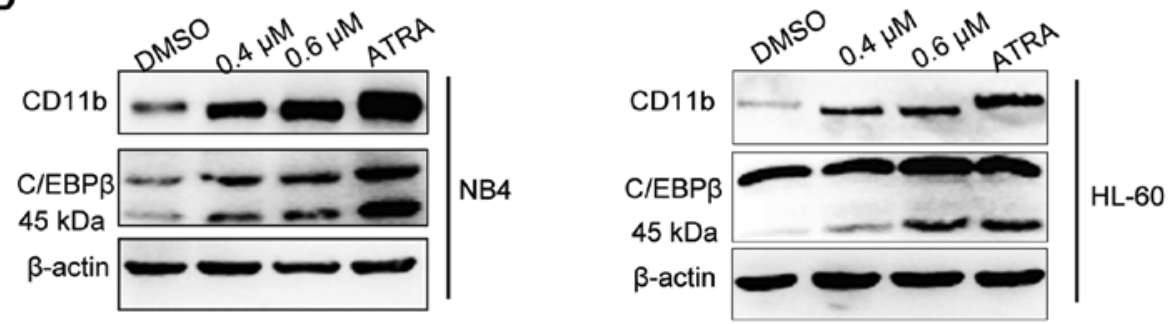

Figure 3. SAL induces cell differentiation in NB4 and HL-60 cells. (A and B) NB4 and HL-60 cells were treated with SAL (0.6 $\mu \mathrm{M})$ or ATRA (1 $\mu \mathrm{M}$, as a positive control) for three days. (A) Then, cell morphology was examined by Wright's staining under a light microscope (magnification, x20 and x100). (B) Differentiation was also assessed by NBT reduction test. A total of 200 cells were counted under a microscope to determine the percentage of NBT-positive cells. Data is expressed as the means \pm SD of three independent experiments. (C) NB4 and HL-60 cells were treated with 0 (DMSO), $0.2,0.4$ and $0.6 \mu \mathrm{M}$ SAL or $1 \mu \mathrm{M}$ ATRA for $72 \mathrm{~h}$, and the percent of differentiated cells was determined by assessing CD11b expression and analyzed by flow cytometry. (D) NB4 and HL-60 cells were treated with 0 (DMSO), 0.4 and $0.6 \mu \mathrm{M} \mathrm{SAL}$ or $1 \mu \mathrm{M}$ ATRA for $72 \mathrm{~h}$, and the expression of differentiation marker CD11b and C/EBP $\beta$ were examined by western blot analysis. Each experiment was repeated at least three times. ${ }^{*} \mathrm{P}<0.05,{ }^{* *} \mathrm{P}<0.01,{ }^{* * * *} \mathrm{P}<0.001 \mathrm{vs}$. the DMSO group, $\mathrm{n}=3$. SAL, salinomycin; ATRA, all-trans retinoic acid; NBT, nitroblue tetrazolium; C/EBP $\beta$, CCAAT/enhancer binding protein $\beta$; DMSO, dimethyl sulphoxide. 
A

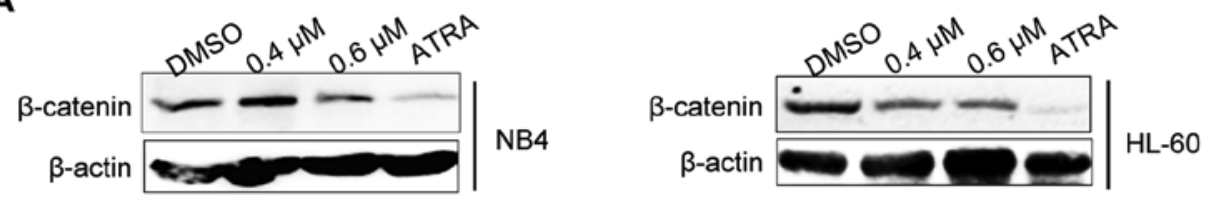

$\mathbf{B}$
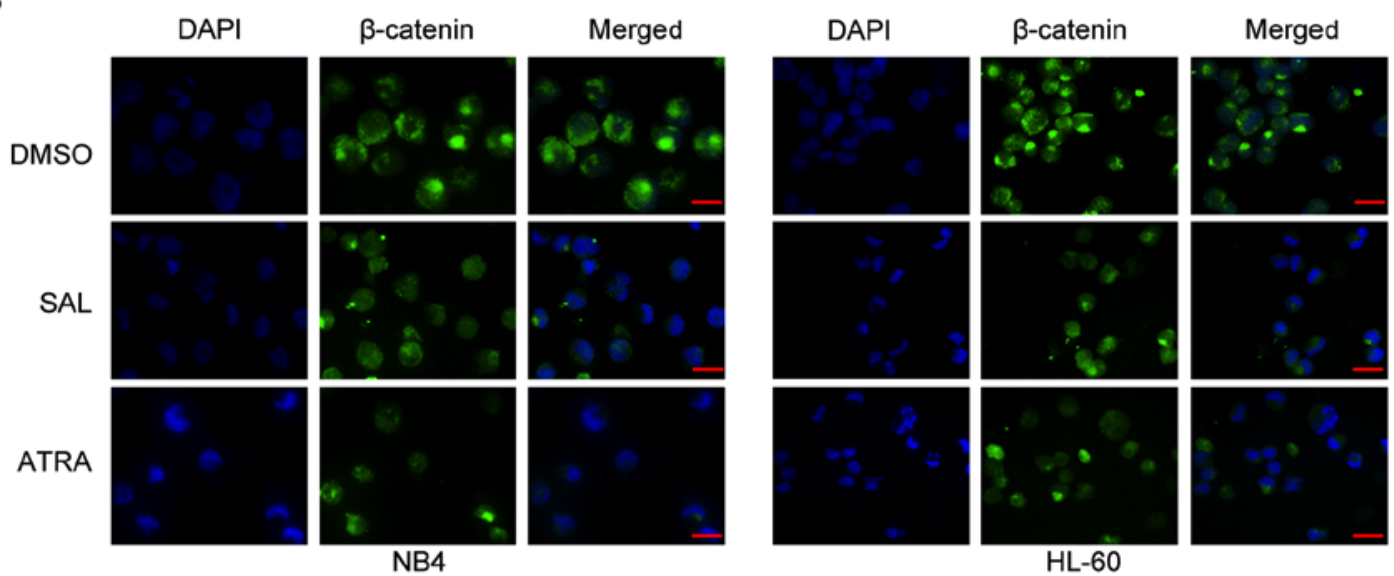

$\mathrm{HL}-60$
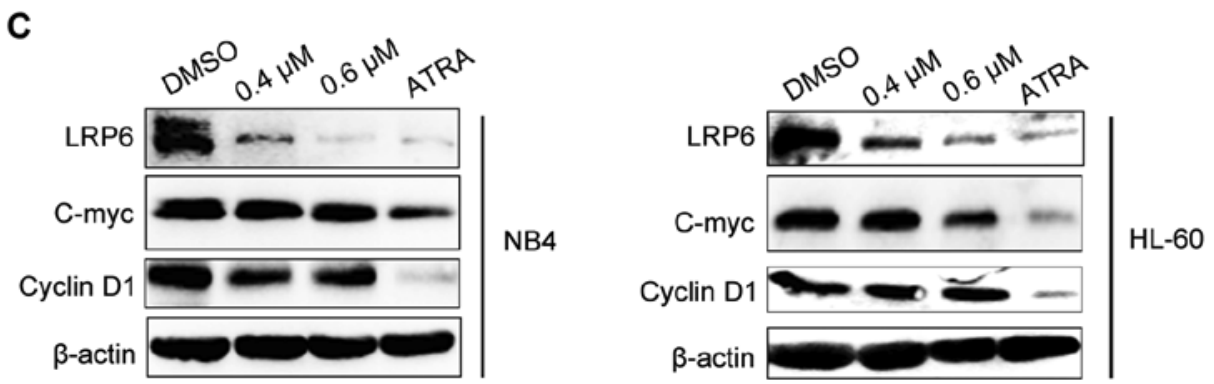

Figure 4. SAL suppresses canonical Wnt signaling in NB4 and HL-60 cells. (A) NB4 and HL-60 cells were treated with different concentrations of SAL or ATRA for $72 \mathrm{~h}$. $\beta$-catenin protein levels were determined by western blot analysis. (B) The expression level and subcellular localization of $\beta$-catenin were examined by immunofluorescence microscopy as described in Materials and methods (magnification, x40). (C) The levels of LRP6, C-myc, cyclin D1 were examined by western blotting. Each experiment was repeated at least three times. SAL, salinomycin; ATRA, all-trans retinoic acid; DMSO, dimethyl sulphoxide.

Wright-Giemsa staining. Morphological analysis revealed that undifferentiated control (DMSO-treated) cells were predominantly promyelocytes with round and large nuclei, whereas cells treated with SAL or ATRA displayed morphological features of cell differentiation, such as a smaller nucleus pattern, cytoplasmic enlargement, lower nuclear/cytoplasmic ratio (Fig. 3A). The percentage of mature NB4 cells increased from $1.5 \%$ among control cells (treated with DMSO) to 39.5 and $74.5 \%$ among cells treated with $0.6 \mu \mathrm{M}$ SAL or $1 \mu \mathrm{M}$ ATRA, respectively. The percentage of mature HL-60 cells increased from $4.0 \%$ among control cells (treated with DMSO) to 30.5 and $63.0 \%$ among cells treated with $0.6 \mu \mathrm{M}$ SAL or $1 \mu \mathrm{M}$ ATRA, respectively. These morphological data were further confirmed by the results of NBT testing. NBT-positive cells significantly increased after treatment with SAL for $72 \mathrm{~h}$ (Fig. 3B). Cell differentiation was further confirmed by detecting the expression of CD11b, a surface myeloid differentiation marker, by performing flow cytometric and western blot analyses. As shown in Fig. 3C, SAL or ATRA treatment significantly increased the percentage of CD11b-positive cells in a dose-dependent manner. Results of western blot analysis also revealed that CD11b expression increased after SAL or ATRA treatment. Previous studies have demonstrated that CCAAT/enhancer binding protein $\beta(\mathrm{C} / \mathrm{EBP} \beta)$ plays a crucial role in myeloid differentiation (30). In the present study, we found that SAL also enhanced C/EBP $\beta$ expression (Fig. 3D). Thus, these results revealed that SAL effectively induced leukemic-cell differentiation.

Salinomycin inhibits Wnt/ $\beta$-catenin signaling. Since activation of canonical Wnt signaling resulted in low ability of cell differentiation (28), we explored whether canonical Wnt signaling was involved in SAL-induced cell differentiation. $\beta$-catenin is the central molecule involved in canonical Wnt signaling, therefore we evaluated $\beta$-catenin expression in NB4 and HL-60 cells treated with SAL $(0.4$ and $0.6 \mu \mathrm{M})$ or ATRA $(1 \mu \mathrm{M})$ for three days by performing western blotting. We found that the total $\beta$-catenin level was decreased after SAL or ATRA treatment for $72 \mathrm{~h}$ (Fig. 4A). It has been revealed that after stabilization and accumulation, $\beta$-catenin translocates into the nucleus and binds transcription factors belonging to T-cell factor/lymphoid enhancer factor (TCF/LEF) family to 
A

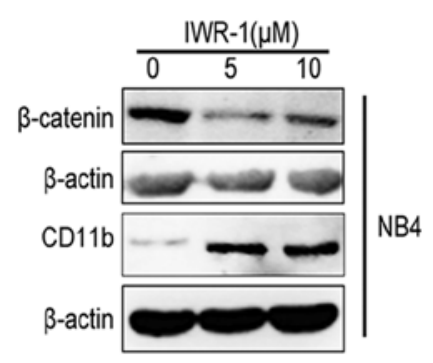

B

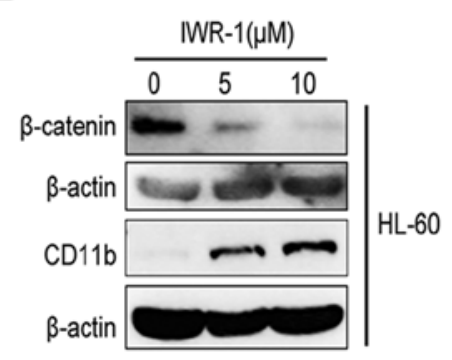

C

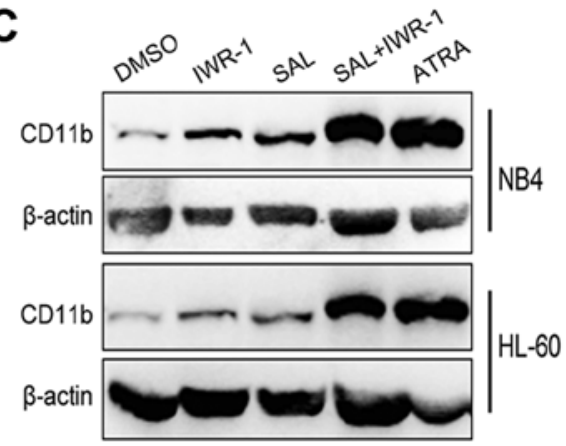

D
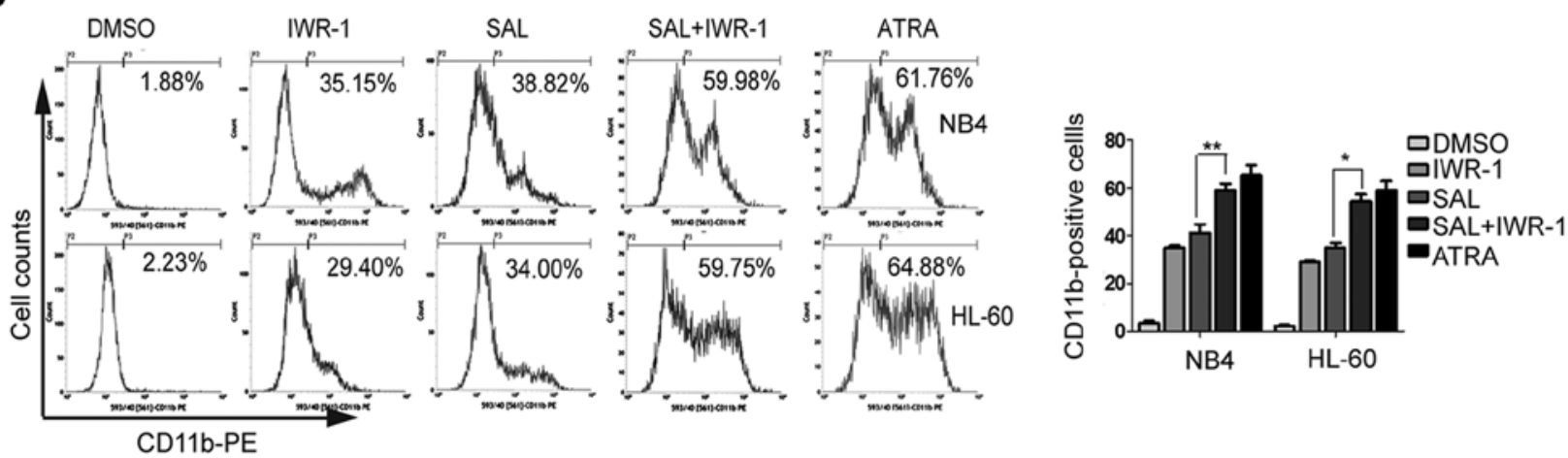

Figure 5. IWR-1 enhances cell differentiation induced by SAL. (A and B) IWR-1 induced cell differentiation. (A) NB4 and (B) HL-60 cells were treated with 0 (DMSO), 5 and $10 \mu$ M IWR-1 for three days, and the expression of $\beta$-catenin and differentiation marker CD11b was assessed by western blotting. (C and D) NB4 and HL-60 cells were treated for three days with IWR-1 $(10 \mu \mathrm{M})$, SAL $(0.4 \mu \mathrm{M})$ or both or ATRA $(1 \mu \mathrm{M}$, as a positive control), and then the cell surface marker CD11b was determined using (C) western blotting and (D) flow cytometry. Each experiment was repeated at least three times. SAL, salinomycin; ATRA, all-trans retinoic acid; DMSO, dimethyl sulphoxide. ${ }^{*} \mathrm{P}<0.05,{ }^{* *} \mathrm{P}<0.01$ vs. the DMSO group.

stimulate the expression of target genes such as cyclin D1 and C-myc (31). Therefore, we investigated the subcellular localization of $\beta$-catenin using immunofluorescence assay. As shown in Fig. 4B, $\beta$-catenin preferentially accumulated in the nucleus of the control (DMSO-treated) cells. SAL or ATRA treatment decreased $\beta$-catenin levels in both the nucleus and cytoplasm. Furthermore, the expression of LRP6, C-myc and cyclin D1 also decreased after treatment with SAL or ATRA for $72 \mathrm{~h}$ (Fig. 4C). These data indicated that Wnt/ $\beta$-catenin signaling was involved in SAL or ATRA induced-cell differentiation.

IWR-1, a Wnt inhibitor, promotes salinomycin-induced cell differentiation. NB4 and HL-60 were treated with Wnt inhibitor IWR-1 (5 or $10 \mu \mathrm{M})$ for $72 \mathrm{~h}$. We observed that IWR-1 treatment decreased $\beta$-catenin expression in NB4 (Fig. 5A) and HL-60 (Fig. 5B) cells. Next, we evaluated whether IWR-1 also induced cell differentiation. As shown in Fig. 5A and B, IWR-1 treatment promoted cell differentiation, as indicated by increased CD11b expression. To further determine whether SAL-induced differentiation of leukemic-cells were involved the canonical Wnt signaling, we investigated the effect of combined treatment with SAL and IWR-1 on NB4 and HL-60 cells. For this, NB4 and HL-60 cells were treated with IWR-1 $(10 \mu \mathrm{M})$, SAL $(0.4 \mu \mathrm{M})$ or both or with ATRA $(1 \mu \mathrm{M})$ for three days. We observed that compared with SAL treatment alone, the combination treatment with IWR-1 and SAL enhanced CD11b expression as determined by performing flow cytometric and western blot analyses (Fig. 5C and D). These results indicated that combined treatment with SAL and
IWR-1 increased cell differentiation and that SAL induced cell differentiation by suppressing Wnt signaling.

\section{Discussion}

Although prognosis of patients with APL has significantly improved since the introduction of ATRA and ATO, the current treatment of APL is associated with some issues such as drug toxicity, resistance and relapse. Therefore, it is necessary to determine novel alternative therapeutic strategies to overcome these issues and to improve the outcome of patients with APL. It was determined that SAL is a potential agent for the elimination of LSCs (20). In addition, it was revealed that SAL exerts non-toxic effects on normal peripheral blood cells $(14,16)$. Therefore, SAL may be a potential drug for the treatment of leukemia and we thus further investigated its effect on apoptosis and differentiation in APL cells in this study.

We firstly evaluated the effect of SAL on cell viability and found that SAL significantly inhibited the growth of NB4 and HL-60 cells (Fig. 1), which was consistent with the result of previous studies assessing the effect of SAL on leukemia cell proliferation $(14,16)$. Next, we investigated whether SAL-induced cell death was accompanied by induction of cell apoptosis. Both flow cytometric analysis and morphological changes revealed that SAL effectively induced APL cells apoptosis (Fig. 2A and B). Apoptosis is regulated by two central apoptotic pathways: the extrinsic pathway (death receptor-mediated pathway) and the intrinsic pathway (mitochondrial-mediated pathway). The extrinsic pathway is 
activated via ligation of death receptors on the cell surface membrane leading to activation of caspase- 8 , followed by caspase- 3 . The intrinsic pathway is mediated by different apoptotic stimuli. Most intrinsic signals induce depolarization of the mitochondrial membrane and the release of cytochrome $c$ into the cytoplasm. The release of cytochrome $c$ activates caspase-9. This results in activation of caspase-3, and commitment to cell death. This pathway is regulated by the B-cell lymphoma 2 family of proteins comprised of 25 proand anti-apoptotic members such as Bcl-2 and Bax (32). To determine the apoptotic pathway induced by SAL in NB4 and HL-60 cells, we further evaluated Bcl-2, Bax, cytochrome $c$, caspase-3, -8 and -9 and PARP expression. We found that the expression of Bax/Bcl-2, cytochrome $c$, cleaved caspase-9, cleaved caspase- 3 and cleaved PARP increased following SAL treatment (Fig. 2). However, cleaved caspase-8 was not observed in our study. These results revealed that SAL induced APL cell apoptosis through the intrinsic pathway. Studies have revealed that inhibition of $\mathrm{Wnt} / \beta$-catenin signaling induces apoptosis of leukemic cells $(14,33)$. To determine whether $\beta$-catenin signaling is involved in SAL-induced apoptosis, we detected the levels of some Wnt-related proteins. We found that the expression of LRP6, $\beta$-catenin and C-myc were also reduced after treatment with 0.8 and $1.6 \mu \mathrm{M} \mathrm{SAL}$ (inducing apoptosis; data not shown). Thus, Wnt/ $\beta$-catenin signaling was also involved in SAL-induced apoptosis.

Since APL is characterized by the accumulation of cells blocked in the promyelocytic stage, targeting cell differentiation is an effective therapy for APL. However, little information is available on role of SAL in modulating leukemia cell differentiation. Therefore, we investigated the potential of SAL to induce the differentiation of APL cell lines. We found that cells treated with SAL exhibited typical morphological changes associated with differentiation. Moreover, SAL treatment markedly increased the percentage of NBT-positive and CD11b-positive cells and protein levels of CD11b and C/EBP $\beta$ (Fig. 3). These results indicated that SAL effectively induced leukemia cell differentiation.

Deregulation of Wnt signaling plays a critical role in the pathogenesis of various types of cancers including AML (34). Moreover, recent studies have revealed that Wnt/ $\beta$-catenin signaling is associated with leukemia cell differentiation (27-29). Therefore, we hypothesized that cell differentiation induced by SAL involves the inhibition of $\mathrm{Wnt} / \beta$-catenin signaling. $\beta$-catenin is at the core of $\mathrm{Wnt} / \beta$-catenin signaling. In the absence of Wnts, cytoplasmic $\beta$-catenin is targeted for ubiquitination and proteasomal degradation and is maintained at a low level. However, the presence of Wnts which bind to Frizzled (Fzd) receptors and lipoprotein receptor-related protein 5/6 (LRP5/6) leads to the formation of the Wnt/Fzd/ LRP5/6 complex on the cell surface. This leads to stabilization of cytosolic $\beta$-catenin, which then translocates into the nucleus to bind to transcription factors of the TCF/LEF family and stimulates the expression of target genes such as cyclin D1 and C-myc $(31,35,36)$. Results of western blot analysis performed in the present study revealed that SAL blocked $\beta$-catenin, C-myc and cyclin D1 expression (Fig. 4). Immunofluorescence analysis revealed that the $\beta$-catenin level was decreased in both the nucleus and cytoplasm of SAL- or ATRA-treated NB4 and HL-60 cells. These results indicated that SAL blocked Wnt/ $\beta$-catenin signaling in NB4 and HL-60 cells. This was consistent with a previous study which revealed that SAL inhibited LRP6, a co-receptor for Wnt ligands and activated $W n t / \beta$-catenin signaling, thus inhibiting $W n t / \beta$ catenin signaling in breast and prostate cancer cells (37). The present study revealed that the LRP6 level was also reduced in SAL-treated NB4 and HL-60 cells (Fig. 4C). To further confirm whether cell differentiation induced by SAL was associated with blocking Wnt/ $\beta$-catenin signaling, we further determined the effect of IWR-1, another Wnt inhibitor (38), on NB4 and HL-60 cells. We found that IWR-1 also enhanced CD11b expression (Fig. 5B). Moreover, compared with SAL treatment alone, the combination treatment with SAL and IWR-1 synergistically triggered the differentiation of NB4 and HL-60 cells (Fig. 5C and D). Collectively, these results indicated that SAL induced leukemia cell differentiation by inhibiting Wnt/ $\beta$-catenin signaling.

Autophagy is a well-known cellular process that plays an important role in the regulation of leukemia cell differentiation. It was previously reported that a high $\beta$-catenin level inhibited autophagy, thus decreasing the differentiation of AML cells (39), and autophagy was upregulated during ATRA-mediated APL cell differentiation (40). Recent studies have revealed that autophagy plays a vital role in regulating PML-RAR $\alpha$ degradation by $\mathrm{p} 62 / \mathrm{SQSTM} 1$ and APL cell differentiation (41). Notably, a recent study revealed that SAL upregulated p62/SQSTM1 expression and activated an autophagic response in AML cell lines (16). Thus, these findings indicated that autophagy may be involved in SAL-induced cell differentiation. However, additional studies are needed to investigate the effect of autophagy on SAL-mediated cell differentiation. In addition, a previous study demonstrated that SAL activated the Toll-like receptor pathway in AML cells (16). Activation of Toll-like receptor pathways has been revealed to promote differentiation and growth inhibition in AML cells (42). Therefore, cell differentiation induced by SAL may be related to Toll-like receptor pathways.

In summary, we found that SAL effectively inhibited the proliferation and induced the apoptosis of NB4 and HL-60 cells. To the best of our knowledge, this is the first study to reveal that SAL induced the differentiation of APL cells, possibly by blocking $W n t / \beta$-catenin signaling. Our results provide a foundation to broaden the clinical application of SAL which may be a promising agent for treatment of APL or other AML types. Further studies are warranted to investigate the combination of ATRA and SAL on APL cells.

\section{Acknowledgements}

Not applicable.

\section{Funding}

The present study was supported by the Natural Science Foundation Project of CQ CSTC (grant no. 2011BA5037).

\section{Availability of data and materials}

The datasets used during the present study are available from the corresponding author upon reasonable request. 


\section{Authors' contributions}

YZ and BZL conceived and designed the study. YZ, LL, SFY, MC, LWL, ZLS, CLX, LGG and TX performed the experiments. YZ wrote the paper. YZ, LZ and BZL reviewed and edited the manuscript. All authors read and approved the manuscript and agree to be accountable for all aspects of the research in ensuring that the accuracy or integrity of any part of the work are appropriately investigated and resolved.

\section{Ethics approval and consent to participate}

Not applicable.

\section{Patient consent for publication}

Not applicable.

\section{Competing interests}

The authors declare that they have no competing interests.

\section{References}

1. Lafage-Pochitaloff M, Alcalay M, Brunel V, Longo L, Sainty D, Simonetti J, Birg F and Pelicci PG: Acute promyelocytic leukemia cases with nonreciprocal PML/RARa or RARa/PML fusion genes. Blood 85: 1169-1174, 1995.

2. Rodeghiero F and Castaman G: The pathophysiology and treatment of hemorrhagic syndrome of acute promyelocytic leukemia. Leukemia 8 (Suppl 2): S20-S26, 1994.

3. Wang ZY and Chen Z: Acute promyelocytic leukemia: From highly fatal to highly curable. Blood 111: 2505-2515, 2008.

4. Wang ZY: Mechanism of action of all-trans retinoic acid and arsenic trioxide in the treatment of acute promyelocytic leukemia. Gan To Kagaku Ryoho 29 (Suppl 1): 214-218, 2002.

5. Tallman MS: Treatment of relapsed or refractory acute promyelocytic leukemia. Best Pract Res Clin Haematol 20: 57-65, 2007

6. Tomita A, Kiyoi H and Naoe T: Mechanisms of action and resistance to all-trans retinoic acid (ATRA) and arsenic trioxide (As2O 3) in acute promyelocytic leukemia. Int J Hematol 97: 717-725, 2013

7. Sanz MA and Montesinos P: How we prevent and treat differentiation syndrome in patients with acute promyelocytic leukemia. Blood 123: 2777-2782, 2014.

8. Butaye P, Devriese LA and Haesebrouck F: Antimicrobial growth promoters used in animal feed: Effects of less well known antibiotics on gram-positive bacteria. Clin Microbiol Rev 16: 175-188, 2003.

9. Gupta PB, Onder TT, Jiang G, Tao K, Kuperwasser C, Weinberg RA and Lander ES: Identification of selective inhibitors of cancer stem cells by high-throughput screening. Cell 138: 645-659, 2009.

10. Xiao Z, Sperl B, Ullrich A and Knyazev P: Metformin and salinomycin as the best combination for the eradication of NSCLC monolayer cells and their alveospheres (cancer stem cells) irrespective of EGFR, KRAS, EML4/ALK and LKB1 status. Oncotarget 5: 12877-12890, 2014.

11. Li T, Liu X, Shen Q, Yang W, Huo Z, Liu Q, Jiao H and Chen J: Salinomycin exerts anti-angiogenic and anti-tumorigenic activities by inhibiting vascular endothelial growth factor receptor 2-mediated angiogenesis. Oncotarget 7: 26580-26592, 2016.

12. Mirkheshti N, Park S, Jiang S, Cropper J, Werner SL, Song CS and Chatterjee B: Dual targeting of androgen receptor and mTORC1 by salinomycin in prostate cancer. Oncotarget 7 : 62240-62254, 2016.

13. Xipell E, Gonzalez-Huarriz M, Martinez de Irujo JJ, GarcíaGarzón A, Lang FF, Jiang H, Fueyo J, Gomez-Manzano C and Alonso MM: Salinomycin induced ROS results in abortive autophagy and leads to regulated necrosis in glioblastoma. Oncotarget 7: 30626-30641, 2016.
14. Lu D, Choi MY, Yu J, Castro JE, Kipps TJ and Carson DA: Salinomycin inhibits Wnt signaling and selectively induces apoptosis in chronic lymphocytic leukemia cells. Proc Natl Acad Sci USA 108: 13253-13257, 2011

15. Niwa AM, D Epiro GF, Marques LA, Semprebon SC, Sartori D, Ribeiro LR and Mantovani MS: Salinomycin efficiency assessment in non-tumor (HB4a) and tumor (MCF-7) human breast cells. Naunyn Schmiedebergs Arch Pharmacol 389: 557-571, 2016.

16. Roulston GD, Burt CL, Kettyle LM, Matchett KB, Keenan HL, Mulgrew NM, Ramsey JM, Dougan C, McKiernan J, Grishagin IV, et al: Low-dose salinomycin induces anti-leukemic responses in AML and MLL. Oncotarget 7: 73448-73461, 2016.

17. Singh A and Settleman J: EMT, cancer stem cells and drug resistance: An emerging axis of evil in the war on cancer. Oncogene 29: 4741-4751, 2010.

18. Mao J, Fan S, Ma W, Fan P, Wang B, Zhang J, Wang H, Tang B, Zhang Q, Yu X, et al: Roles of Wnt/ $\beta$-catenin signaling in the gastric cancer stem cells proliferation and salinomycin treatment. Cell Death Dis 5: e1039, 2014.

19. Lee HG, Shin SJ and Chung HW: Salinomycin reduces stemness and induces apoptosis on human ovarian cancer stem cell. J Gynecol Oncol 28: e14, 2017.

20. Fuchs D, Daniel V, Sadeghi M, Opelz G and Naujokat C: Salinomycin overcomes ABC transporter-mediated multidrug and apoptosis resistance in human leukemia stem cell-like KG-1a cells. Biochem Biophys Res Commun 394: 1098-1104, 2010.

21. Zou ZZ, Nie PP, Li YW, Hou BX, Rui-Li, Shi XP, Ma ZK, Han BW and Luo XY: Synergistic induction of apoptosis by salinomycin and gefitinib through lysosomal and mitochondrial dependent pathway overcomes gefitinib resistance in colorectal cancer. Oncotarget 8: 22414-22432, 2017.

22. Hermawan A, Wagner E and Roidl A: Consecutive salinomycin treatment reduces doxorubicin resistance of breast tumor cells by diminishing drug efflux pump expression and activity. Oncol Rep 35: 1732-1740, 2016.

23. Li R, Dong T, Hu C, Lu J, Dai J and Liu P: Salinomycin repressed the epithelial-mesenchymal transition of epithelial ovarian cancer cells via downregulating $\mathrm{Wnt} / \beta$-catenin pathway. Onco Targets Ther 10: 1317-1325, 2017.

24. Clevers H: Wnt/beta-catenin signaling in development and disease. Cell 127: 469-480, 2006.

25. Simon M, Grandage VL, Linch DC and Khwaja A: Constitutive activation of the Wnt/beta-catenin signalling pathway in acute myeloid leukaemia. Oncogene 24: 2410-2420, 2005.

26. Luis TC, Ichii M, Brugman MH, Kincade P and Staal FJ: Wnt signaling strength regulates normal hematopoiesis and its deregulation is involved in leukemia development. Leukemia 26: 414-421, 2012.

27. Zang S, Liu N, Wang H, Wald DN, Shao N, Zhang J, Ma D, Ji C and Tse W: Wnt signaling is involved in 6-benzylthioinosineinduced AML cell differentiation. BMC Cancer 14: 886, 2014.

28. Sheng Y, Ju W, Huang Y, Li J, Ozer H, Qiao X and Qian Z: Activation of wnt $/ \beta$-catenin signaling blocks monocytemacrophage differentiation through antagonizing PU.1-targeted gene transcription. Leukemia 30: 2106-2109, 2016.

29. Gandillet A, Park S, Lassailly F, Griessinger E, Vargaftig J, Filby A, Lister TA and Bonnet D: Heterogeneous sensitivity of human acute myeloid leukemia to $\beta$-catenin down-modulation. Leukemia 25: 770-780, 2011.

30. Lee SC, Kim OH, Lee SK and Kim SJ: IWR-1 inhibits epithelial-mesenchymal transition of colorectal cancer cells through suppressing Wnt/ $\beta$-catenin signaling as well as survivin expression. Oncotarget 6: 27146-27159, 2015

31. McCubrey JA, Steelman LS, Bertrand FE, Davis NM, Abrams SL, Montalto G, D'Assoro AB, Libra M, Nicoletti F, Maestro R, et al: Multifaceted roles of GSK-3 and Wnt/ $\beta$-catenin in hematopoiesis and leukemogenesis: Opportunities for therapeutic intervention. Leukemia 28: 15-33, 2014.

32. Xu G and Shi Y: Apoptosis signaling pathways and lymphocyte homeostasis. Cell Res 17: 759-771, 2007.

33. Chen Y, Liu ZH, Xia J, Li XP, Li KQ, Xiong W, Li J and Chen DL: 20(S)-ginsenoside Rh2 inhibits the proliferation and induces the apoptosis of KG-1a cells through the Wnt $/ \beta$-catenin signaling pathway. Oncol Rep 36: 137-146, 2016.

34. Staal FJ, Famili F, Garcia Perez L and Pike-Overzet K: Aberrant Wnt Signaling in Leukemia. Cancers (Basel) 8: 8, 2016.

35. King TD, Suto MJ and Li Y: The Wnt/ $\beta$-catenin signaling pathway: A potential therapeutic target in the treatment of triple negative breast cancer. J Cell Biochem 113: 13-18, 2012. 
36. Ahmadzadeh A, Norozi F, Shahrabi S, Shahjahani M and Saki N: Wnt/ $\beta$-catenin signaling in bone marrow niche. Cell Tissue Res 363: 321-335, 2016.

37. Lu W and Li Y: Salinomycin suppresses LRP6 expression and inhibits both $\mathrm{Wnt} / \beta$-catenin and mTORC1 signaling in breast and prostate cancer cells. J Cell Biochem 115: 1799-1807, 2014.

38. Chen B, Dodge ME, Tang W, Lu J, Ma Z, Fan CW, Wei S Hao W, Kilgore J, Williams NS, et al: Small molecule-mediated disruption of Wnt-dependent signaling in tissue regeneration and cancer. Nat Chem Biol 5: 100-107, 2009.

39. Kühn K, Cott C, Bohler S, Aigal S, Zheng S, Villringer S, Imberty A, Claudinon J and Römer W: The interplay of autophagy and $\beta$-Catenin signaling regulates differentiation in acute myeloid leukemia. Cell Death Discov 1: 15031, 2015.
40. Orfali N, O'Donovan TR, Nyhan MJ, Britschgi A, Tschan MP, Cahill MR, Mongan NP, Gudas LJ and McKenna SL: Induction of autophagy is a key component of all-trans-retinoic acidinduced differentiation in leukemia cells and a potential target for pharmacologic modulation. Exp Hematol 43: 781-793, 2015.

41. Wang Z, Cao L, Kang R, Yang M, Liu L, Zhao Y, Yu Y, Xie M, Yin X, Livesey KM, et al: Autophagy regulates myeloid cell differentiation by p62/SQSTM1-mediated degradation of PML-RAR $\alpha$ oncoprotein. Autophagy 7: 401-411, 2011.

42. Ignatz-Hoover JJ, Wang H, Moreton SA, Chakrabarti A, Agarwal MK, Sun K, Gupta K and Wald DN: The role of TLR8 signaling in acute myeloid leukemia differentiation. Leukemia 29: 918-926, 2015. 